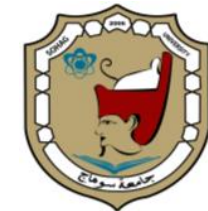

Sohag University

\title{
Association of common variants in the IGF2BP2 gene with type 2 diabetes
}

\section{Wael Abd El-Hameid Ali ${ }^{1}$, Sahar Abd El-Atty Sharaf ${ }^{2}$, Elham O Hamid $^{1,3}$ and Ali Taha $\mathrm{Ali}^{4}$}

1. Clinical and Chemical Pathology Department, Faculty of Medicine, Sohag University.

2. Clinical and Chemical Pathology Department, Faculty of Medicine, Cairo University.

3. Applied Medical Science, Al-Ghad International University, Dammam College, Kingdom of Saudi Arabia.

4. Internal Medicine Department, Faculty of Medicine, Sohag University.

\begin{abstract}
:
Background: Several studies had addressed the relationship between the common variants in the insulin-like binding protein 2 (IGF2BP2) gene and type 2 diabetes (T2DM). In this study, we aimed to find the association of IGF2BP2 variants rs4402960 and rs 1470579 with type 2 DM in Egyptian people.

Methods: The study included genotyping of SNPs in (IGF2BP2) gene (rs 1470579) and (rs4402960) variants using Real-time PCR in 50 types two DM patients and 50 control subjects.
\end{abstract}

Results: Using logistic regression of this additive model of SNP rs4402960, the presence of homozygous variant allele (TT) genotype carries a 3-fold risk of DM with an odds ratio $(\mathrm{OR})$ of $3.28(\mathrm{p}=0.095)$, and homozygous variant allele $(\mathrm{CC})$ genotype of SNP rs1470579 carries a 5-fold risk of DM with OR of $5.44(\mathrm{p}=0.011)$. Using the dominant model, the two IGF2BP2 variants carry a high risk of DM. Meanwhile, using the recessive model only SNP rs1470579 carries a risk near 3-fold risk of DM.

Conclusions: This study shows that insulin-like binding protein 2 susceptibility variants rs4402960 and rs1470579 are associated with type two DM in Egypt.

Keywords: Diabetes, IGF2BP2, PCR, and SNP.

\section{Introduction:}

Diabetes mellitus type 2 (T2DM) is characterized by high blood glucose levels due to defective secretion of insulin and impairment of its action. T2DM normally starts with resistance to insulin where the cell fails to respond properly to insulin. The capacity to produce insulin is lost meanwhile the need for it grows. Insulin is important for glucose metabolism and blood glucose regulation. Insulin is critical for the metabolism of blood glucose, and the insulin pathway involves genes that are essential in determining the genetic causes of T2DM (1).

IGF2BP2 is a member of the insulinlike growth factor 2 (IGF2) mRNA-binding protein 2 (IGF2BP2) family of IGF2 mRNA-binding proteins that are essential in the embryogenesis and development of the pancreas. IGF2BP2 can also control IGF2 transcription, which is essential for the production and activity of insulin (2). 
Insulin secretion, which is essential for glucose metabolism, is regulated by the IGF2BP2 gene. rs4402960 and rs1470579 that we investigated are the most widely studied polymorphisms of this gene and yielded both solid and weak or no associations with T2DM $(3,4)$.

\section{Study objectives:}

Evaluating the association between the rs4402960 and rs1470579 variants of IGF2BP2 and type2 DM in the Egyptian people.

\section{Methodology of the study: \\ Patients:}

This research was enrolled 100 persons divided as fifty T2DM patients seeking medical service at the diabetes clinic of Sohag University Hospital in the period from December 2016 to August 2017, and fifty healthy volunteers who were age and gender-matched to the $1^{\text {st }}$ group.

\section{Inclusion criteria:}

1. T2DM patients according to $\mathrm{WHO}$ criteria and ADA (ADA,2019).

2. Egyptian patients aging more than 30 years.

3. Persons who can and willing to provide consent and fulfill the requirements of the protocol.

participants were divided into 2 groups:

- Group I (Diabetic)

- Group II (Control)

All participants were subjected to:

1. Inquiries about medical history

2. Clinical examination

3. Laboratory tests:

- Blood samples were taken from patients and controls as follow:

- Eight $\mathrm{ml}$ of venous blood was obtained by clean venipuncture and then divided into 3 portions:

o Two ml on EDTA (Ethylene Diamine Tetra acetic Acid) for PCR.
- Two ml on EDTA (Ethylene Diamine Tetra acetic Acid) for glycated hemoglobin (HbA1c).

- Four $\mathrm{ml}$ on the plain tube for separation of serum for blood chemistry.

- Random Urine sample: for $\mathrm{A} / \mathrm{C}$ ratio estimation.

- The following investigations were also performed for all participants:

- Fasting and 2 hours postprandial blood glucose level

○ Glycated hemoglobin (HbA1c)

o cholesterol, triglycerides, HDL-c, VLDL-c, and LDL-c.

- Albumin /Creatinine ratio

○ A genomic study using real-time PCR technique.

- Genetic studies (SNP genotyping): Identification of the following:

○ SNP ID rs 1470579.

- SNP ID rs4402960.

- Real-time PCR technique the following steps were done:

- DNA extraction from whole blood using QIAamp DNA blood MinikitQiagen (from Hoffmann-La Roche AG ) according to the protocol proposed by Fabris et al., 2011.

- The extracted DNA was then amplified corresponding to the method proposed by Kristiansen et al., 2003.

o Real-time PCR was used for the genotyping.

\section{Statistical analysis:}

Analysis was done using SPSS $®$ Statistics version 22. As applicable, numerical data were presented as mean and standard deviation or median and range. Frequency and percentage were used to express qualitative results. Pearson's Chi-square test or Fisher's exact test was used to examining the relationship between qualitative variables. For quantitative data, comparison between two groups was done using either Student ttest for normally distributed data or Mann-Whitney test (non-parametric t-test) for not normally distributed data. 
The Kruskal-Wallis test (non-parametric ANOVA) was used for comparison between the 3 groups. Each SNP was tested for Hardy-Weinberg equilibrium using the Chi-square goodness-of-fit test. Logistic regression analysis was used to calculate risk after controlling for age and triglyceride as covariates. The odds ratio (OR) with its $95 \%$ confidence interval $(\mathrm{CI})$ was used for risk estimation. All tests were two-tailed. A p-value $<0.05$ was believed to be significant.

\section{Results:}

This research was done on two groups: the Diabetic $(n=50)$ including patients with T2DM and the Control $(n=50)$ including healthy volunteers.

Males were slightly more common among cases (48\%) compared to controls (32\%), with a p-value of 0.102 , but the diabetic group was statistically older than the control group $(48.1 \pm 6.1$ years for cases, $38.3 \pm 5.4$ for controls, $p$ less than 0.001).
The diabetic patients had statistically higher triglycerides levels, very lowdensity lipoprotein, and albumin/createnine ratio. Cholesterol levels were relatively higher in the diabetic group, but the difference was not statistically significant $(\mathrm{p}=0.080)$. After categorization of lipid profile items, it was shown that the Control group has a significantly high proportion of individuals with high-risk levels of HDL $(\leq 35 \mathrm{mg} / \mathrm{dL})$. The entire control group had a normal $\mathrm{A} / \mathrm{C}$ ratio, while $96 \%$ of the diabetic group had a high ratio ( $\mathrm{p}<0.001)$.

The IGF2BP2 variant rs4402960 mutation was more common in the diabetic persons (78\%; 34 homozygous) compared to the volunteers $(66 \%$; 16 homozygous), but the difference shows only a trend towards significance $(\mathrm{p}=0.095)$ Fig (1). On the other hand, SNP rs1470579 was more frequent in the diabetic group (74\%; 34\% homozygous) compared to the controls $(50 \%$; $12 \%$ homozygous) with a p-value of $0.011 \mathrm{Fig}(2)$.

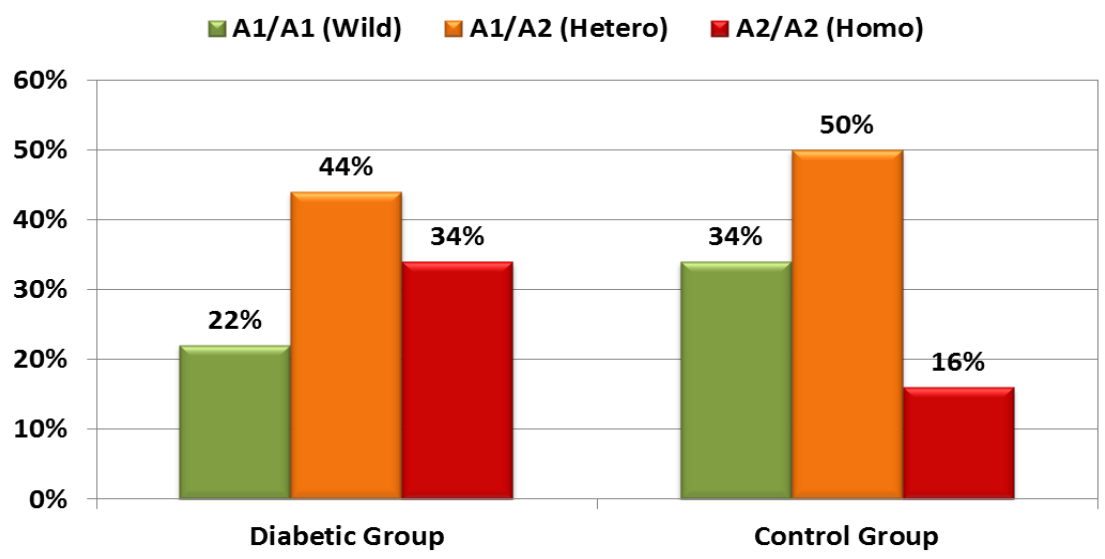

Figure 1: SNP rs4402960 variants in diabetic and control groups 


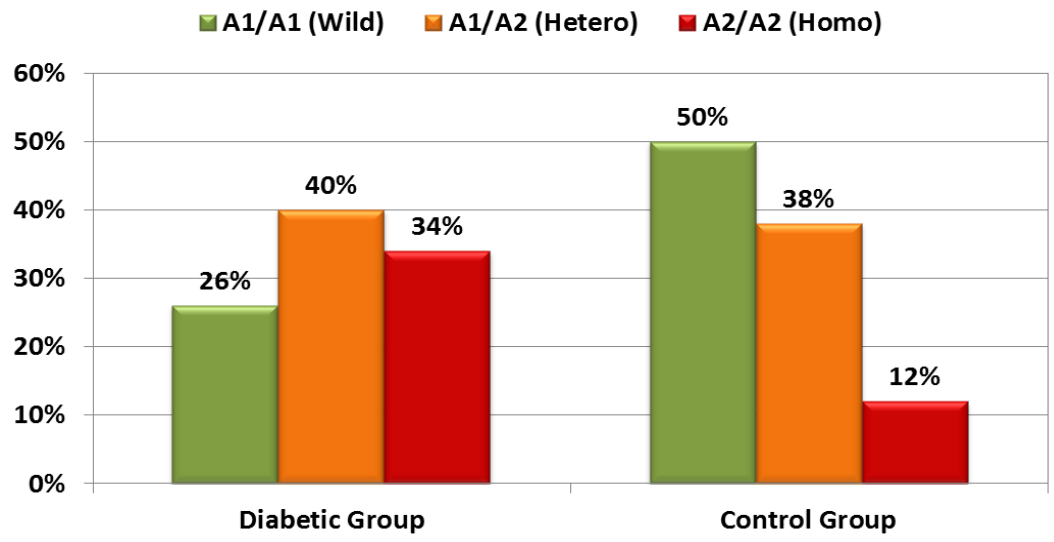

Figure 2: SNP rs1470579 variants in diabetic and control groups

Using logistic regression of this additive model of SNP rs4402960, the presence of homozygous T/T genotype carries a 3-fold risk of DM with an odds ratio (OR) of 3.28 (95\% CI: 1.1-10.2). Also, homozygous C/C genotype of SNP rs1470579 carries a 5-fold risk of DM with OR of 5.44 (95\% CI: 1.717.2).
Using the dominant model (Homozygous variant allele vs. Homozygous reference allele + Heterozygous, the two IGF2BP2 variants carry a high risk of DM (Table 16). Meanwhile, using the recessive model (Heterozygous + Homozygous variant allele vs. Homozygous reference allele), only SNP rs1470579 carries a risk of a near 3 fold risk of DM (Table 1).

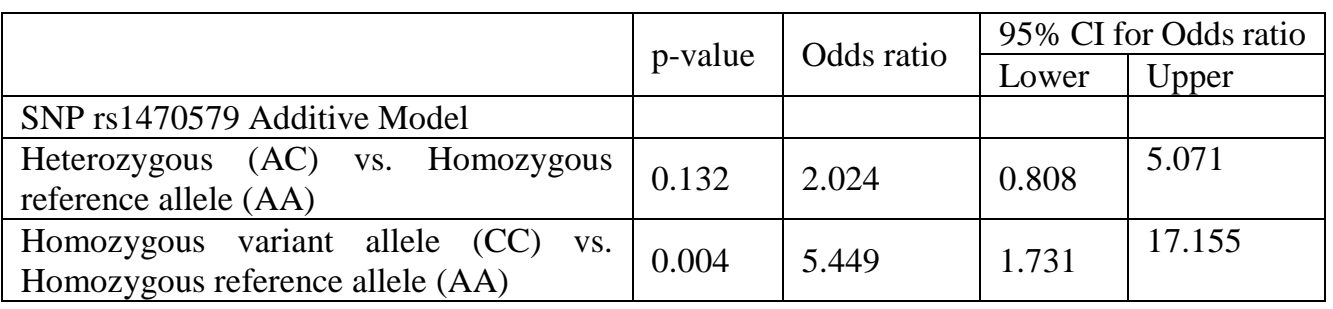

Table 1: Risk estimation of diabetes mellitus with IGF2BP2 variant rs1470579

\begin{tabular}{|c|c|c|c|c|}
\hline & $\begin{array}{l}\text { Diabetic } \\
(\mathrm{n}=50)\end{array}$ & $\begin{array}{l}\text { Control } \\
(\mathrm{n}=50)\end{array}$ & $\mathrm{p}$ value & $\begin{array}{l}\text { Odds ratio } \\
\text { Confidence interval } 95 \%\end{array}$ \\
\hline SNP rs4402960 & & & \multirow{3}{*}{0.038} & \multirow{3}{*}{$\begin{array}{l}2.71 \\
(1.04-7.04)\end{array}$} \\
\hline Homozygous variant (TT) & $17(34 \%)$ & $8(16 \%)$ & & \\
\hline $\begin{array}{l}\text { Homozygous reference }(\mathrm{GG})+ \\
\text { Heterozygous }(\mathrm{GT})\end{array}$ & $33(66 \%)$ & $42(84 \%)$ & & \\
\hline SNP rs1470579 & & & \multirow{3}{*}{0.009} & \multirow{3}{*}{$\begin{array}{l}3.78 \\
(1.34-10.63)\end{array}$} \\
\hline Homozygous variant (CC) & $17(34 \%)$ & $6(12 \%)$ & & \\
\hline $\begin{array}{l}\text { Homozygous reference (AA) }+ \\
\text { Heterozygous }(\mathrm{AC})\end{array}$ & $33(66 \%)$ & $44(88 \%)$ & & \\
\hline
\end{tabular}

Table 2: Risk estimation of diabetes mellitus with the dominant model of IGF2BP2 variant rs1470579

\section{Discussion:}

The characteristic feature of type 2 diabetes mellitus is high levels of serum glucose due to impairment of insulin production and activity. T2DM normally starts with resistance to insulin, in which the cells fail to respond appropriately to insulin. The pancreas gradually loses its ability to generate insulin while the 
need for it increases. Insulin is important for glucose metabolism and blood glucose regulation. Insulin is critical for the metabolism of blood glucose, and the insulin pathway involves genes that are essential in determining the genetic causes of T2DM (5).

IGF2BP2 is a member of the insulinlike growth factor 2 (IGF2) mRNAbinding protein 2 (IGF2BP2) family of IGF2 mRNA-binding proteins that are essential in the embryogenesis and development of the pancreas. IGF2BP2 can also control IGF2 transcription, which is essential for the production and activity of insulin (6).

Insulin secretion, which is essential for glucose metabolism, is regulated by the IGF2BP2 gene. rs4402960 and rs1470579 that we investigated are the most widely studied polymorphisms of this gene and yielded both solid and weak or no associations with T2DM (7).

This research is to find out the possible involvement of IGF2BP2 variants rs4402960 and rs1470579 with T2DM in Egyptian people.

This research included two groups: The Diabetic (50 patients) including patients with T2DM and the Control (50 healthy) including nondiabetic individuals. The two groups were comparable regarding sex without significant difference $(p=0.102)$. aging is a risk factor for T2DM. The effect presented by the probability allele altered with aging (8). In this study, patients in the diabetic group were older than those in the Control Group ( $\mathrm{p}<0.001$ ). Similar to our results, Rao et al. found rs1470579 to be significantly correlated with type $2 \mathrm{DM}$ in the mean age of more than sixty studies, but not in studies with a mean age of fifty-five. There may be a connection between aging and IGF2BP2. Further research with a bigger sample size and prospective studies is required to verify the connection (9). pancreatic development in animals is affected by IGF2BP2 which is a signa- ling molecule for growth and insulin (10).

As expected, we found that the Diabetic patients had considerably higher levels of HbA1c, and fasting, and 2-hour postprandial blood glucose compared to the Control group. Huang et al. found that T2DM patients who carried the C allele of rs1470579 had higher levels of FPG, postprandial serum insulin, and TC compared to AA carriers (11).

On hyperglycaemic clamps, Rs4402960 reduces the first step of glucose-induced insulin production. (12). Subsequent research in other populations revealed similar findings (13).

Thus, it could be said that the IGF2BP2 variants have an important role in the control of beta-cell activity (11).

Given That the 2 SNPs are located in the $50-\mathrm{kb}$ region of intron 2 , diabetespredisposing variants likely affect IGF2BP2 expression control (14).

Moreover, SNPs could be related to adjacent variants that have a role on larger non-coding transcripts, micro RNAs, etc. (15). Also, rs4402960 and rs1470579 may be proxy markers rather than a true operating variant. It has been reported that the diacylglycerol kinase g-1 (DGKG) located closed to IGF2$\mathrm{BP} 2$, is associated with regulation of the metabolism $(16,17)$. Therefore, further functional research into the pathophysiological mechanisms of IGF2BP2 is needed.

In this study, the Diabetic Group had greater levels of Triglycerides, very lowdensity lipoprotein, and albumin/creatinine ratio. Cholesterol levels were relatively higher in the Diabetic Group, but without a statistically important difference $(p=0.080)$. After categorization of lipid profile items, it was shown that the Control group has a significantly higher proportion of individuals with high-risk levels of HDL $(\leq 35$ $\mathrm{mg} / \mathrm{dL}$ ). The entire Control group had a normal A/C ratio, while $96 \%$ of the Diabetic Group had a high ratio ( $\mathrm{p}<$ 
0.001). The Diabetic Group had a higher level of IGF2BP2 variant $G$ allele of rs4402960 mutation than the Control Group, but the difference show$\mathrm{s}$ only a trend towards significance $(\mathrm{p}=$ 0.095). On the other hand, the SNP A allele of rs1470579 was significantly more frequent in the Diabetic Group ( $\mathrm{p}$ $=0.011$ ). When we used logistic regression of this additive model of SNP rs4402960, the presence of homogenous A2/A2 genotype carries a 3-fold risk of DM with an odds ratio (OR) of 3.28 (95\% CI: 1.1-10.2), and homogenous A2/A2 genotype of SNP rs1470579 carries a 5-fold risk of DM with OR of 5.44 (95\% CI: 1.7-17.2). Using the dominant model (A2/A2 homo vs. A1/A1+A1/A2), the two IGF2BP2 variants carry a high risk of DM. Meanwhile, using the recessive model (A1/A2+A2/A2 vs. A1/A1), only SNP rs 1470579 carries a risk a nearly 3 -fold risk of DM.

In line with our results, Nemr et al. found the strongest association at rs4402960, followed by rs1470579, with calculated odds ratio (OR) values of 1.60 and 1.36 , respectively. To minimize the possibility of population bias in associated studies, they enrolled Arabic Lebanese subjects, and both type 2 DM patients and nondiabetic control volunteers were from the same area (18).

For rs4402960, the relationship was stronger than found in the original GWAS in European people such as Danish (6), French (7), and Austrians (19), and Chinese populations (20). Other studies on Norwegians (21), Israeli (19), Moroccans (19), and Pima Indians (22) founded no relation of rs4402960 with type $2 \mathrm{DM}$. The lack of a significant relation of rs4402960 in these studies may not be due to the lack of power and thus it is likely attached to differences in ethnic/racial settings. Larger sample sizes in other ethnic groups are crucial to offi- cially reproduce the association of rs4402960 IGFBP2 variant with type 2 DM. The effects of rs 1470579 on the danger of T2DM are conflicting among different ethnic groups. For example, Cauchi reported a marginal association (OR, 1.17 ; 95\% CI, 1.07-1.27) between rs1470579 and T2DM in a French population (19), while Duesing et al. described that there was no significant link in other French people (7).

Furthermore, a case-control study by Rao et al. revealed that rs4402960 and rs1470579 variants linked with the probability of type $2 \mathrm{DM}$ under the rec-essive genetic models $(p<0.05)$, without Statistically significant difference in other models. With the findings of a casecontrol report, a meta-analysis of thirtysix Asian papers was performed (9).

Benrahma et al. observed a significant correlation between IGF2BP2 rs4402960 polymorphism and diabetes under additive 2 and recessive models (23), but in a previous report in a Moroccan population, Saxena et al. stated that there was no association of this SNP with diabetes $(\mathrm{p}=0.22)(24)$.

Additionally, the $\mathrm{T}$ allele frequency was $37.66 \%$ in controls, like the frequency found in South Asians (32\%), Europeans $(31 \%)$, and East Asians (28\%); the maximum incidence $(49 \%)$ was observed in an African population $(3,23)$.

Moreover, several types of research tried to clarify the connection between IGF2BP2 rs4402960 variants and diabetes. The IGF2BP2 gene, also known as IMP2, is a member of a family of mRNA-binding proteins (IMP1, IMP2, and IMP3) that are implicated in the embryogenesis of the pancreatic tissues (15).

\section{Conclusion:}

The IGF2BP2 (rs4402960) (TT) homozygous variant allele genotype carried a 3 -fold risk factor for the occurrence of type 2 diabetes in Egyptian patients while the homozygous variant allele (CC) 
genotype of SNP rs 1470579 carries a 5 fold risk of DM.

\section{Recommendations:}

Serum Insulin level and HOMA equation should be estimated

Studying the association of (rs4402960) and (rs1470579) variants of IGF2BP2 gene with the risk of other diseases e.g. coronary heart disease, retinopathy, glaucoma, and risk of cancer e.g. leukemia, gastric and colorectal cancer, breast cancer... etc

Further studies in larger numbers of patients are necessary to establish the role of IGF2BP2 variants.

A multicentric study should be performed that will help in increasing the statistical power by alleviating possible differences of studied subjects, as the ethnic background, gene-environment interaction including economic, nutritional status, and the duration of diabetes. Future studying of (rs4402960) and (rs1470579) variants could be done together with other variants of IGF2BP2 genes.

\section{References:}

1. Perry JR, Voight BF, Yengo L, Amin N, Dupuis J, Ganser M, et al. Stratifying type 2 diabetes cases by BMI identifies genetic risk variants in LAMA1 and enrichment for risk variants in lean compared to obese cases. PLoS Genet. 2012;8(5):e1002741.

2. Christiansen JS, Liebl A, Davidson JA, Ligthelm RJ, Halimi S. Mid- and highratio premix insulin analogs: potential treatment options for patients with type 2 diabetes in need of greater postprandial blood glucose control. Diabetes Obes Metab. 2010;12(2):10514.

3. Nfor ON, Ndzinisa NB, Tsai MH, Hsiao $\mathrm{CH}$, Liaw YP. Interactive Effect of IGF2BP2 rs4402960 Variant, Smoking and Type 2 Diabetes. Diabetes Metab Syndr Obes. 2020;13:5097-102.

4. Liu SP, Qian WJ. Association between IGF2BP2 rs4402960 polymorphism and risk of type 2 diabetes mellitus: the need for clarification of data in a recent metaanalysis. Arch Med Res. 2012;43(5):411; author reply 2-3.

5. Parra EJ, Below JE, Krithika S, Valladares A, Barta JL, Cox NJ, et al. Genome-wide association study of type 2 diabetes in a sample from Mexico City and a meta-analysis of a MexicanAmerican sample from Starr County, Texas. Diabetologia. 2011;54(8):203846.

6. Grarup N, Rose CS, Andersson EA, Andersen G, Nielsen AL, Albrechtsen $A$, et al. Studies of association of variants near the HHEX, CDKN2A/B, and IGF2BP2 genes with type 2 diabetes and impaired insulin release in 10,705 Danish subjects: validation and extension of genome-wide association studies. Diabetes. 2007;56(12):3105-11.

7. Duesing K, Fatemifar G, Charpentier G, Marre M, Ticket J, Hercberg S, et al. Evaluation of the association of IGF2BP2 variants with type 2 diabetes in French Caucasians. Diabetes. 2008;57(7):1992-6.

8. Ding D, Chong S, Jalaluddin B, Comino E, Bauman AE. Risk factors of incident type 2-diabetes mellitus over a 3-year follow-up: Results from a large Australian sample. Diabetes Res Clin Pract. 2015;108(2):306-15.

9. Rao P, Wang H, Fang H, Gao Q, Zhang $\mathrm{J}$, Song $\mathrm{M}$, et al. Association between IGF2BP2 Polymorphisms and Type 2 Diabetes Mellitus: A Case-Control Study and Meta-Analysis. Int J Environ Res Public Health. 2016;13(6).

10.Cunha MR, da Silva ME, Machado HA, Fukui RT, Correa MR, Santos RF, et al. The effects of metformin and glibenclamide on glucose metabolism, counter-regulatory hormones and cardiovascular responses in women with Type 2 diabetes during exercise of moderate intensity. Diabet Med. 2007;24(6):592-9.

11.Huang Q, Yin JY, Dai XP, Pei Q, Dong $M$, Zhou ZG, et al. IGF2BP2 variations influence repaglinide response and risk of type 2 diabetes in Chinese population. Acta Pharmacol Sin. 2010;31(6):709-17.

12. Groenewoud MJ, Dekker JM, Fritsche A, Reiling E, Nijpels G, Heine RJ, et al. 
Variants of CDKAL1 and IGF2BP2 affect first-phase insulin secretion during hyperglycaemic clamps. Diabetologia. 2008;51(9):1659-63.

13.Horikoshi M, Hara K, Ito C, Nagai R, Froguel P, Kadowaki T. A genetic variation of the transcription factor 7like 2 gene is associated with risk of type 2 diabetes in the Japanese population. Diabetologia. 2007;50(4):747-51.

14. Scott RA, Scott LJ, Magi R, Marullo L, Gaulton KJ, Kaakinen M, et al. An Expanded Genome-Wide Association Study of Type 2 Diabetes in Europeans. Diabetes. 2017;66(11):2888-902.

15.Christiansen J, Kolte AM, Hansen T, Nielsen FC. IGF2 mRNA-binding protein 2: biological function and putative role in type 2 diabetes. J Mol Endocrinol. 2009;43(5):187-95.

16. Wang J, Chen L, Qiang P. The role of IGF2BP2, an m6A reader gene, in human metabolic diseases and cancers. Cancer Cell Int. 2021;21(1):99.

17.Rodriguez S, Eiriksdottir G, Gaunt TR, Harris TB, Launer LJ, Gudnason V, et al. IGF2BP1, IGF2BP2 and IGF2BP3 genotype, haplotype and genetic model studies in metabolic syndrome traits and diabetes. Growth Horm IGF Res. 2010;20(4):310-8.

18.Nemr R, Echtay A, Dashti EA, Almawi AW, Al-Busaidi AS, Keleshian SH, et al. Strong association of common variants in the IGF2BP2 gene with type 2 diabetes in Lebanese Arabs. Diabetes Res Clin Pract. 2012;96(2):225-9.

19. Cauchi S, Meyre D, Durand E, Proenca C, Marre M, Hadjadj S, et al. Postgenome-wide association studies of novel genes associated with type 2 diabetes show gene-gene interaction and high predictive value. PLoS One. 2008;3(5):e2031.

20.Han X, Luo Y, Ren Q, Zhang X, Wang $F$, Sun $X$, et al. Implication of genetic variants near SLC30A8, HHEX, CDKAL1, CDKN2A/B, IGF2BP2, FTO, TCF2, KCNQ1, and WFS1 in type 2 diabetes in a Chinese population. BMC Med Genet. 2010;11:81.

21.Hertel JK, Johansson S, Raeder H, Midthjell K, Lyssenko V, Groop L, et al. Genetic analysis of recently identified type 2 diabetes loci in 1,638 unselected patients with type 2 diabetes and 1,858 control participants from a Norwegian population-based cohort (the HUNT study). Diabetologia. 2008;51(6):971-7.

22.Rong R, Hanson RL, Ortiz D, Wiedrich C, Kobes S, Knowler WC, et al. Association analysis of variation in/near FTO, CDKAL1, SLC30A8, HHEX, EXT2, IGF2BP2, LOC387761, and CDKN2B with type 2 diabetes and related quantitative traits in Pima Indians. Diabetes. 2009;58(2):478-88.

23. Benrahma H, Charoute H, Lasram K, Boulouiz R, Atig RK, Fakiri M, et al. Association analysis of IGF2BP2, KCNJ11, and CDKAL1 polymorphisms with type 2 diabetes mellitus in a Moroccan population: a case-control study and meta-analysis. Biochem Genet. 2014;52(9-10):430-42.

24.Saxena M, Agrawal CG, Srivastava N, Banerjee M. Interleukin-6 (IL-6)-597 $\mathrm{A} / \mathrm{G} \quad(\mathrm{rs} 1800797) \quad \& \quad-174 \quad \mathrm{G} / \mathrm{C}$ (rs1800795) gene polymorphisms in type 2 diabetes. Indian $\mathrm{J}$ Med Res. 2014;140(1):60-8. 